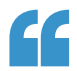

The validation of hits identified in the screening revealed several unexpected mechanisms of resistance that would not have been detected by genetic analysis

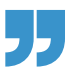

\title{
THERAPEUTICS
}

\section{Winning combination}

Therapies targeting specific genetic changes that drive tumour growth can achieve remission with fewer side effects than other less-targeted therapies; however, this approach is hindered by the development of resistance. To identify effective therapeutic combinations that can circumvent resistance, Crystal et al. have developed a screening platform that tests cell cultures grown directly from patient tumour samples against a panel of drugs that target pathways involved in cell growth and survival.

The authors established 20 cell cultures from patients with nonsmall cell lung cancer whose disease had progressed after treatment with epidermal growth factor receptor (EGFR) or anaplastic lymphoma kinase (ALK) tyrosine kinase inhibitors. Each cell culture was tested against a panel of 76 targeted drugs, both alone and in combination with the first-line drug to which the tumour had become resistant. To confirm the effectiveness of the strategy, the authors also tested the approach in five established cell lines with a known mechanism of resistance developed in vitro. In all five cell lines, the screen accurately identified drugs that effectively targeted the known bypass mechanism of resistance. The approach was then used in a panel of 55 resistant cell lines with unknown mechanisms of resistance, which included the cell cultures established from patient biopsy samples. In these cells, the combination screen included next-generation inhibitors of the gatekeeper mutations (EGFR or ALK) as a primary drug and a second drug. At least one effective combination was identified in 45 of the 55 lines tested. Importantly, treatment with single agents was not effective, emphasizing the importance of developing combination therapies.

In a cell culture derived from a patient who had become resistant to several EGFR inhibitors, the screen identified MET inhibitors as 'hits' and, indeed, treatment of these cells with the MET inhibitor crizonitib in an ample range of concentrations re-sensitized these cells to treatment with the EGFR inhibitor gefitinib. The screen identified other hits that restored sensitivity to EGFR inhibition, such as inhibitors of fibroblast growth factor receptor 3 (FGFR3), as well as aurora kinase and polo-like kinase inhibitors.

The validation of hits identified in the screening revealed several unexpected mechanisms of resistance that would not have been detected by genetic analysis. For example, several $A L K$-positive tumours were re-sensitized by drugs that inhibit SRC, even when no SRC-related mutations were present. Further evidence indicated that inhibiting ALK led to activation of SRC-mediated pathways that promoted resistance, and that combining ALK and SRC inhibitors as first-line therapy might delay the emergence of resistance.

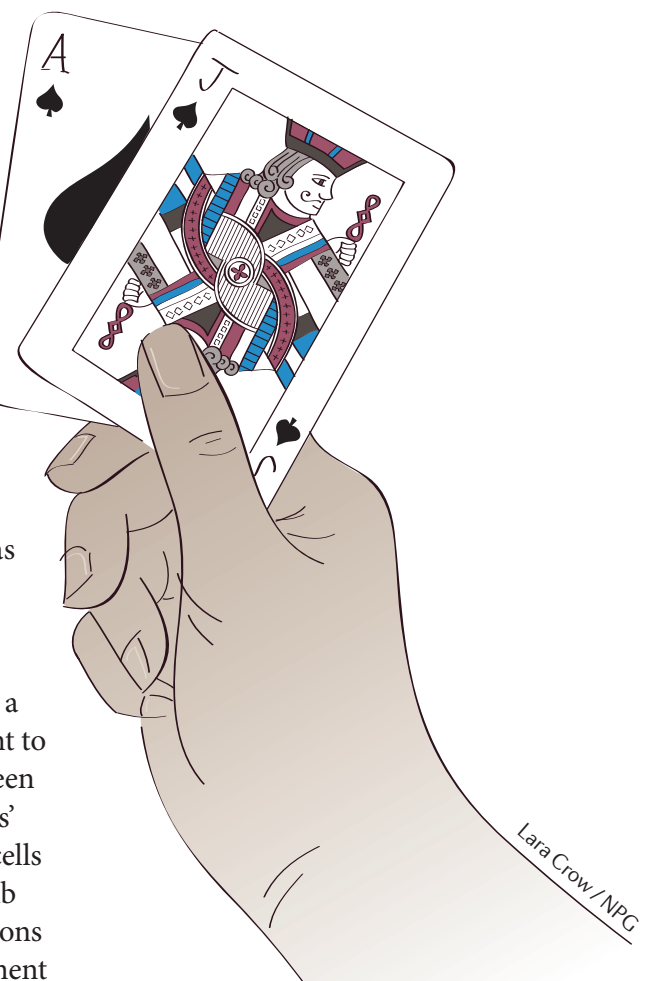

The efficacy of this combination was demonstrated in vivo in several patient-derived xenograft models.

Finally, the authors point out that establishment of the patientderived cell cultures to carry out the screening could take 2-6 months, a time frame that would make this approach less than ideal as a routine diagnostic test. Hopefully, the development of new techniques might enable screens to be carried out on viable cells obtained within weeks of a biopsy.

M. Teresa Villanueva

ORIGINAL RESEARCH PAPER Crystal, A. S. et al. Patient-derived models of acquired resistance can identify effective drug combinations for cancer. Science http://dx.doi.org/10.1126/ science.1254721 (2014) 